\title{
SYNCHROTRON RADIATION IN DIRECTIONS CLOSE TO MAGNETIC-FIELD LINES
}

\author{
K. C. WESTFOLD
}

Monash University, Australia

\begin{abstract}
Astrophys. Space Sci.). It is characteristic of the radiation from a particle of mass $m$ bearing a charge $e$ moving with ultrarelativistic velocity $\beta c$ in a magnetic field of induction $\mathbf{B}_{0}$ that the bulk of the emission is confined to a small cone of directions within $O(\xi)$ of the direction of motion $\tau$ which makes a constant angle $\alpha$ with $\mathbf{B}_{0}$, and that for any direction $\mathbf{n}$ at an angle $\theta$ with $\mathbf{B}_{0}$ such emission is observed as harmonics of the fundamental frequency $f_{B_{0}} \xi / a$, where $f_{B_{0}}$ is the nonrelativistic cyclotron frequency $e B_{0} / 2 \pi m, \xi=\sqrt{ }\left(1-\beta^{2}\right) \ll 1$, and
\end{abstract}

$$
a=1-\beta \cos \alpha \cos \theta \text {. }
$$

It follows that the radiation from a distribution of particles in general appears as a series of lines, broadened with respect to the dependence of the distribution on both pitch angle and energy $E=m c^{2} / \xi$.

In directions away from the field lines, $\sin \theta=O(1), a \simeq \sin ^{2} \theta$, and the bulk of the emission is in the high-order harmonics $n=O\left(\xi^{-3}\right)$. Although the broadening with respect to pitch angle is slight, the observed radiation is quasicontinuous.

In directions close to the field lines, for which $\sin \theta=O(\xi), a \simeq \frac{1}{2}\left(\xi^{2}+\sin ^{2} \alpha+\sin ^{2} \theta\right)=$ $=O\left(\xi^{2}\right)$, so that pitch-angle broadening is significant and there is a significant contribution to the radiation from low-order harmonics. Specifically, if $N(1 / \xi) \mathrm{d}(1 / \xi)$ is the number density of particles in the energy range $(E, E+\mathrm{d} E)$ and $2 \pi \phi(\alpha) \sin \alpha \mathrm{d} \alpha$ is the fraction of 'these in the pitch-angle range $(\alpha, \alpha+\mathrm{d} \alpha)$, the emissivity tensor corresponding to the $n$th harmonic is, to a first approximation, distributed with respect to the frequency $f$ according to the formulae

$$
\begin{aligned}
& \boldsymbol{\eta}_{n}(\mathbf{n})=\int_{0}^{n f_{B_{0}} / \sin \theta} \boldsymbol{\eta}_{n f}(\mathbf{n}) \mathrm{d} f, \\
& \boldsymbol{\eta}_{n f}(\mathbf{n})=\frac{2 \pi n^{2} y^{3}}{f_{B_{0}}} \int_{n y-\sqrt{ }\left(n^{2} y^{2}-\sin ^{2} \theta\right)} N\left(\frac{1}{\xi}\right) \phi(\alpha)\left\langle\mathbf{P}_{n}(\mathbf{n})\right\rangle \mathrm{d} \xi,
\end{aligned}
$$

where $y=f_{B_{0}} / f$ and is of magnitude $O(\xi / n)$, and, in terms of base vectors $i_{1}$ along the projection of $B_{0}$ on the plane transverse to $n=i_{3}$ and $i_{2}=i_{3} \times i_{1}$, the emission polarization tensor

$$
\left\langle\mathbf{P}_{n}(\mathbf{n})\right\rangle=\frac{\mu e^{2} c f_{B_{0}}^{2}}{2 n^{2} y^{4} \xi^{2}}\left[\left(\frac{n y \xi-\sin ^{2} \theta}{\sin \theta}\right)^{2} J_{n}^{2}\left(\frac{\sin \alpha \sin \theta}{y \xi}\right) \mathbf{i}_{1} \mathbf{i}_{1} \mp\right.
$$




$$
\begin{aligned}
& \mp_{i} \frac{n y \xi-\sin ^{2} \theta}{\sin \theta} \sin \alpha J_{n}\left(\frac{\sin \alpha \sin \theta}{y \xi}\right) J_{n}^{\prime}\left(\frac{\sin \alpha \sin \theta}{y \xi}\right)\left(\mathbf{i}_{1} \mathbf{i}_{2}-\mathbf{i}_{2} \mathbf{i}_{1}\right)+ \\
& \left.+\sin ^{2} \alpha J_{n}^{\prime 2}\left(\frac{\sin \alpha \sin \theta}{y \xi}\right) \mathbf{i}_{2} \mathbf{i}_{2}\right]
\end{aligned}
$$

according as $\cos \theta_{<}^{>} O$. The pitch angle $\alpha$ is given in terms of $\xi$ and $y$ by the relation

$$
\sin \alpha=\sqrt{ }\left(2 n y \xi-\xi^{2}-\sin ^{2} \theta\right) .
$$

Since the argument of the Bessel functions is $n \times O(1)$, it is not possible to make a further simplification without making further assumptions. For emission in directions close to the field lines it is appropriate to $\operatorname{take} \sin \theta \ll \xi$ and to expand the formulae in powers of $(\sin \theta) / \xi$. Then, for $n=1$, the magnitude of the expression in square brackets is $O\left(\xi^{2}\right)$, and for each subsequent harmonic it is reduced by the factor

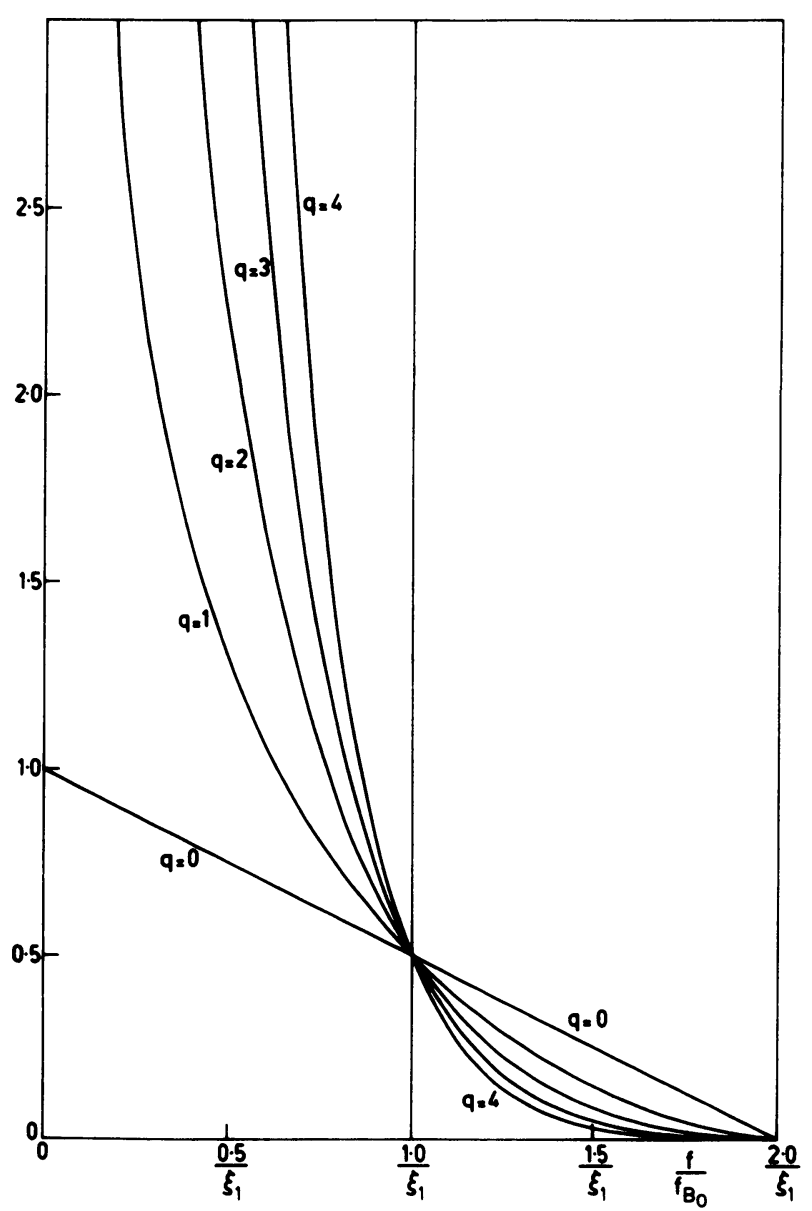

Fig. 1. The emissivity parameter $\eta_{f} /\left(\pi \mathscr{N} \mu e^{2} c f_{B_{0}} a_{q} \xi_{1}^{q+1}\right)$ for monoenergetic particles with pitch-angle distribution $\phi(\alpha)=a_{q} \sin ^{q} \alpha$. 
$O\left(\left(\sin ^{2} \theta\right) / \xi^{2}\right)$. The approximations have been carried through for the first two harmonics, giving formulae for the total emissivity tensor

$$
\boldsymbol{\eta}_{f}(\mathbf{n})=\sum_{n} \boldsymbol{\eta}_{n f}(\mathbf{n})
$$

of consistent accuracy and showing that, contrary to what has previously been reported, the degree of circular polarization is $1+O\left(\left(\sin ^{4} \theta\right) / \xi^{4}\right), \mathrm{RH}$ for $\cos \theta>O$ and $\mathrm{LH}$ for $\cos \theta<0$.

In particular, for a monoenergetic distribution for which $N(1 / \xi)=\mathscr{N} \delta\left(1 / \xi-1 / \xi_{1}\right)$, we find as a first approximation to the emissivity

$$
\eta_{f}(\mathbf{n})=\pi \mathscr{N} \mu e^{2} c f_{B_{0}} \phi\left(\alpha_{1}\right) \xi_{1}\left(1-\frac{\xi_{1} f}{2 f_{B_{0}}}\right),
$$

where the pitch angle $\alpha_{1}$ corresponding to the frequency $f$ is given by

$$
\frac{\sin \alpha_{1}}{\xi_{1}}=\left(\frac{2 f_{B_{0}}}{\xi_{1} f}-1\right)^{1 / 2} \text {. }
$$

A particular case of the dependence of emissivity on pitch-angle distribution is exhibited in Figure 1 where we have taken

$$
\phi(\alpha)=a_{q} \sin ^{q} \alpha,
$$

a distribution which varies from isotropic with respect to velocity when $q=1$ to a concentration of flat helical trajectories about the value $\alpha=\frac{1}{2} \pi$ when $q$ is increased, resulting in reduced emission close to $\theta=0$ and $\pi$. The portion of the curves for which $O \leqslant f / f_{B_{0}}<1 / s \xi_{1}$ correspond to $\left(\sin \alpha_{1}\right) / \xi_{1}>(2 s-1)^{1 / 2}$, and should be ignored when $s$ is so large that the requirement that $\left(\sin \alpha_{1}\right) / \xi_{1}$ should be $O(1)$ is breached. They should all fall sharply to zero at $f / f_{B_{0}}=\frac{1}{2} \xi_{1}$. 